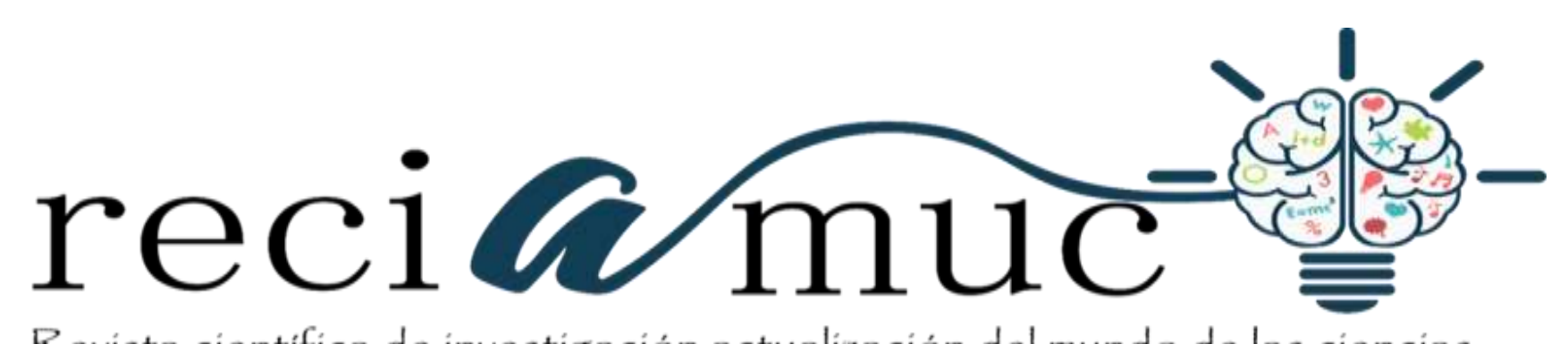

Revista cientifica de investigación actualización del mundo de las ciencias

Ivonne Carolina Recalde Tello a; Marlon Enrique Allauca Yumiseba ${ }^{\text {b; }}$ Jhoanna

Patricia Puruncajas Maza ${ }^{c}$; Gema Adriana Alarcón Medina ${ }^{\mathrm{d}}$

Contraindicaciones de analgesia epidural post cesárea

Post cesárea epidural analgesia contraindications

Revista Científica de Investigación actualización del mundo de las Ciencias. Vol. 3

núm., 2, abril, ISSN: 2588-0748, 2018, pp. 921-932

DOI: $10.26820 /$ reciamuc/3.(2).abril.2019.921-932

URL: http://reciamuc.com/index.php/RECIAMUC/article/view/372

Código UNESCO: 3205 Medicina Interna

Tipo de Investigación: Artículo de Revisión

(C) RECIAMUC; Editorial Saberes del Conocimiento, 2019

Recibido: 15/01/2019

Aceptado: 07/02/2019

Publicado: 01/04/2019

Correspondencia: ivonne_ecua16@hotmail.com

a. Médico; Investigador Independiente; Guayaquil, Ecuador; ivonne ecua16@ hotmail.com

b. Médico; Investigador Independiente; Guayaquil, Ecuador; marlon.allauca@gmail.com

c. Médico; Investigador Independiente; Guayaquil, Ecuador; jhoupm@ hotmail.com

d. Médico; Investigador Independiente; Guayaquil, Ecuador; gema202_@hotmail.com 


\section{Contraindicaciones de analgesia epidural post cesárea}

Vol. 3, núm. 2., (2019)

Ivonne Carolina Recalde Tello; Marlon Enrique Allauca Yumiseba; Jhoanna Patricia Puruncajas Maza; Gema Adriana Alarcón Medina

\section{RESUMEN}

La cesárea es una práctica que va en crecimiento cada año a nivel mundial. Uno de sus síntomas más importantes es el dolor, el cual repercute significativamente en la recuperación de la paciente. La Analgesia Epidural constituye una de las técnicas más usadas y con mayores ventajas para la administración de la analgesia post cesárea, no obstante, no se encuentra exenta de complicaciones. El objetivo fundamental de la presente investigación es plasmar las ventajas de la analgesia epidural post cesárea en contraposición con sus desventajas. El diseño de investigación que se llevó a cabo es de tipo documental o bibliográfico. Dentro de las múltiples ventajas que presenta esta técnica se encuentran: disminución de la morbimortalidad perioperatoria, mejor analgesia, menor sedación, además de movilización rápida del paciente. En contraposición, puede presentar riesgo de depresión respiratoria, infecciones en la piel y tejido subcutáneo, incluso neuroinfección. Asimismo, puede presentarse daño neurológico directo por la colocación del catéter. En conclusión, la investigación con respecto a la técnica es escasa y mayormente se encuentra orientada a los efectos de los fármacos administrados por medio de la misma, en razón de lo cual, la relación riesgo - beneficio se inclina hacia el uso de la técnica.

Palabras claves: Contradicciones; Analgesia; Epidural; Post; Cesárea. 


\title{
Contraindicaciones de analgesia epidural post cesárea
}

Vol. 3, núm. 2., (2019)

Ivonne Carolina Recalde Tello; Marlon Enrique Allauca Yumiseba; Jhoanna Patricia Puruncajas

Maza; Gema Adriana Alarcón Medina

\begin{abstract}
Caesarean section is a practice that is growing every year worldwide. One of its most important symptoms is pain, which has a significant impact on the patient's recovery. Epidural Analgesia is one of the most used techniques and with greater advantages for the administration of postcesarean analgesia, however, it is not without complications. The main objective of the present investigation is to capture the advantages of post-cesarean epidural analgesia in contrast to its disadvantages. The research design that was carried out is documentary or bibliographic. Among the many advantages of this technique are: decreased perioperative morbidity and mortality, better analgesia, less sedation, as well as rapid patient mobilization. In contrast, it may present a risk of respiratory depression, infections of the skin and subcutaneous tissue, including neuroinfection. Also, direct neurological damage may occur due to catheter placement. In conclusion, research regarding the technique is scarce and is mostly oriented towards the effects of the drugs administered through it, because of which, the risk-benefit ratio leans towards the use of the technique.
\end{abstract}

Key words: Contradictions; Analgesia; Epidural; Post; Cesarean. 


\section{Contraindicaciones de analgesia epidural post cesárea}

Vol. 3, núm. 2., (2019)

Ivonne Carolina Recalde Tello; Marlon Enrique Allauca Yumiseba; Jhoanna Patricia Puruncajas Maza; Gema Adriana Alarcón Medina

\section{Introducción.}

En la actualidad, la cesárea es una de las intervenciones quirúrgicas más realizadas a nivel mundial. Cada día sus tasas son más altas, especialmente en países de medios y altos ingresos. La cesárea debe realizarse sólo cuando el parto normal sea imposible, o ponga en riesgo el bienestar y la vida de la madre y el hijo, no obstante, a menudo se llevan a cabo sin ninguna necesidad, que fuera de representar un beneficio puede ocasionar problemas de salud. A nivel mundial, la tasa de cesárea supera la tasa ideal, la cual está establecido por la OMS entre el $10 \%$ y el $15 \%$. Específicamente, en las Américas, esta tasa oscila alrededor del 38,9\%, esto en base a los últimos datos disponibles de 25 países. Se estima que esta cifra puede ser mayor considerando las tasas de cesáreas que se realizan en clínicas del sector privado, a las cuales no se tiene acceso. (Organización Panamericana de la Salud, 2015)

Revisando otras cifras, entre 2006 y 2010, el 15\% de los nacimientos se llevaron a cabo por medio de cesárea. Ubicándose Brasil como el país con mayor alto porcentaje de cesárea con 50\%, México es el segundo con 43\%. En el caso de Centroamérica, el primer lugar lo tiene El Salvador con $25 \%$ y con la más baja tasa se encuentra Honduras con 13\%. Para el año 2012 se reportaron 13.939.455 millones de nacimientos, de los cuales 5.420.236 terminaron en una cesárea. Asimismo, datos parciales del año 2013 señalan que esta cifra sigue en aumento. (Organización Panamericana de la Salud, 2014)

La cesárea conlleva una serie de complicaciones para la madre, incluso para su hijo. Esta intervención es de recuperación mucho más larga que la de un parto normal, además puede originar una fibrosis de la matriz y el aumento de estos riesgos están relacionados con el número de cesáreas 


\section{Contraindicaciones de analgesia epidural post cesárea}

Vol. 3, núm. 2., (2019)

Ivonne Carolina Recalde Tello; Marlon Enrique Allauca Yumiseba; Jhoanna Patricia Puruncajas Maza; Gema Adriana Alarcón Medina

que se practica en una paciente. Incluso, en comparación con los partos normales existe mayor tasa de mortalidad en los partos por cesárea.

Uno de los principales síntomas asociados a la cesárea es el dolor y este repercute de manera significativa en la recuperación de la mujer y en la relación que debe desarrollarse durante las primeras horas postquirúrgicas entre el hijo y su madre.

El manejo del dolor postoperatorio en las pacientes de cesárea es un elemento importante, el cual se realiza en base a técnicas analgésicas que tratan de minimizar los efectos secundarios de este tratamiento, que son de buena calidad y su duración es prolongada, todo ello con la finalidad de proporcionar alivio del dolor de manera rápida, una mejor recuperación para la madre y disminución en los costos de atención hospitalaria. (Lorca \& Calventos, 2013)

En este sentido, la analgesia se puede definir como "la pérdida o modulación de la percepción del dolor. Puede ser 1) local y afectar sólo una pequeña área del cuerpo, 2) regional y afectar una porción más amplia del cuerpo o 3) sistémica”. Las técnicas analgésicas son variadas, entre ellas tenemos el uso de la hipnosis, los medicamentos sistémicos, los fármacos regionales o por inhalación, entre otros. La Analgesia denota estados en donde es exclusiva la participación de la modulación perceptiva del dolor. A diferencia de la Anestesia, que incluye estados en los que se pierde la conciencia mental, además de la percepción de otras sensaciones, perspectiva desde la cual, la analgesia podría considerarse como un componente de la anestesia. (McDonald, BiingJaw, \& Wing-Fai, 2014) 


\section{Contraindicaciones de analgesia epidural post cesárea}

Vol. 3, núm. 2., (2019)

Ivonne Carolina Recalde Tello; Marlon Enrique Allauca Yumiseba; Jhoanna Patricia Puruncajas Maza; Gema Adriana Alarcón Medina

La analgesia por medio de fármacos es la más usada con una amplia gama de vías de administración, que buscan calmar el dolor minimizando las complicaciones asociadas al uso del medicamento y de la técnica. Una de las más usadas en todo el mundo, en el manejo del dolor postoperatorio de la cesárea, es la vía epidural.

La analgesia epidural, es la técnica mediante la cual se inyectan analgésicos en el espacio epidural, el cual se ubica en el conducto vertebral, fuera del líquido cefalorraquídeo. Durante el proceso se introduce un tubo delgado y largo llamado catéter, entre dos vértebras en la zona de la espalda, por medio del cual se administra la medicación. (Mayo Clinic, 2018)

A pesar de ser la técnica más usada para el control del dolor que se presenta después de una cesárea por sus mínimos riesgos, y de la creencia en todo el mundo de ser una técnica inocua, no está exenta de presentar algunas complicaciones o riesgos.

El objetivo de la presente investigación es plasmar las desventajas de la analgesia epidural usada en el periodo de postcesárea en contraposición a las ventajas.

\section{Materiales y Métodos}

Se realizó una revisión bibliográfica de fuentes de información relacionadas con las desventajas de la analgesia epidural postcesárea en contraposición a sus ventajas. El período de búsqueda se realizó entre el año 2012 y el año 2019, a los fines de mantener el principio de actualidad y diferenciar la investigación por su contenido más novedoso. 


\section{Contraindicaciones de analgesia epidural post cesárea}

Vol. 3, núm. 2., (2019)

Ivonne Carolina Recalde Tello; Marlon Enrique Allauca Yumiseba; Jhoanna Patricia Puruncajas Maza; Gema Adriana Alarcón Medina

Para Ríos (2017) la investigación documental “[...] recurre a la consulta de documentos para obtener sus resultados". (p. 81). En base a lo anterior, el presente estudio se clasifica por su metodología como una investigación de tipo documental por la fuente usada para su realización.

\section{Resultados}

Mille Loera (2018) La analgesia epidural postoperatoria es una técnica usada con mucha frecuencia que "requiere de planificación y trabajo en equipo, aunque la analgesia peridural ofrece beneficios bien documentados en pacientes seleccionados, algunos cirujanos la consideran excesiva y suelen tener sus propias opiniones al respecto". Dentro de sus múltiples ventajas se encuentran la disminución de la morbimortalidad perioperatoria, una menor incidencia general de complicaciones, asimismo la tasa de infecciones pulmonares es menor, al igual que el tiempo necesario de intubación, aunado a los costos hospitalarios. La técnica ofrece una mejor analgesia, una menor sedación, además de movilización rápida del paciente con respecto a otros procedimientos. En contraposición, puede presentar riesgo de depresión respiratoria, una infección en la piel y el tejido subcutáneo hasta neuroinfección y, en el peor de los casos, daño neurológico directo por la colocación del catéter o debido a los fármacos utilizados. (p. 5-8)

Ramos, Ferrer, Mojica, \& González (2017) explican que el tema del uso de medicamentos no opioides coadyuvantes a nivel neuroaxial, es importante y de gran interés, por cuanto disminuye la dosis de opioide y con ello sus indeseados efectos secundarios. Asimismo, estudios han demostrado que este medicamento, en ese nivel, puede prolongar el efecto analgésico, sin embargo, existen efectos secundarios importantes. Una de las técnicas para hacer llegar el medicamento a nivel neuroaxial y esperar menos complicaciones, es la administración epidural, 


\section{Contraindicaciones de analgesia epidural post cesárea}

Vol. 3, núm. 2., (2019)

Ivonne Carolina Recalde Tello; Marlon Enrique Allauca Yumiseba; Jhoanna Patricia Puruncajas Maza; Gema Adriana Alarcón Medina

con la cual se disminuye la dosis requerida de analgésico local, además de reducir el prurito y no aumenta considerablemente el riesgo de hipotensión, de sedación o de efectos adversos en el feto. Una de las dificultades o desventajas es que tiene un coste elevado con relación a otras técnicas consideradas también efectivas. (p. 330)

El catéter epidural presenta múltiples beneficios para la paciente en la movilización requerida para la atención de su hijo en las primeras horas, no obstante, no se encuentra exenta de riesgos o complicaciones. La mayoría de los problemas relacionados con los catéteres epidurales pueden ser problemas mecánicos, aquellos casos donde el catéter no funciona, el catéter se encuentra en posición y funciona, pero el analgésico no es el adecuado para el paciente y migración del catéter, cuando no se encuentra en el espacio epidural. (Mille Loera, 2018, p. 7,8)

Muchos autores coinciden en que la mayoría de las desventajas del uso de la analgesia epidural como tratamiento del dolor postoperatorio en cesáreas se asocian principalmente al analgésico o conjunto de medicamentos administrados y no a la técnica en sí.

En concordancia, Garza Hinojosa (2012) refiere que son variadas las ventajas para el uso de la analgesia epidural post-operatoria tales como proporcionar una "analgesia de mejor calidad que con analgésicos por otras vías, además, disminuye la morbilidad post-operatoria. (cardiovascular, respiratoria, tromboembólica e infecciosa". No obstante, las desventajas presentadas por tipo de fármaco usado por esta vía son las siguientes: 1. Anti-inflamatorios no esteroideos (AINE’S): Pacientes con enfermedad ácido-péptica, corren riesgo de ulceración de la mucosa y sangrado. 


\section{Contraindicaciones de analgesia epidural post cesárea}

Vol. 3, núm. 2., (2019)

Ivonne Carolina Recalde Tello; Marlon Enrique Allauca Yumiseba; Jhoanna Patricia Puruncajas Maza; Gema Adriana Alarcón Medina

Pacientes con enfermedad renal previa, hipovolémicos, deshidratados, cirróticos o con bajo gasto, pueden ser desencadenantes de que su condición empeore. El uso en forma crónica, aumenta el sangrado trans. y post-operatorio. En el caso de los anestésicos locales administrados por vía epidural se han presentado en algunos casos hipotensión, náusea, bradicardia, parestesia pasajera, retención urinaria y fiebre.

Uno de los tipos de analgesia epidural más modernos en la actualidad es la controlada por el paciente. Para Sukhyanti, Kirti, \& Bharti (2017) la analgesia epidural controlada por el paciente en el periodo post cesárea es mucho más efectiva. Asimismo, refieren que la adición de fentanilo a levobupivacaína, provoca mayor mareo y prurito, además de menos parestesia que con analgesia epidural controlada por el paciente con levobupivacaína sola. (p. 203)

Asimismo, es importante destacar que la mayoría de los efectos adversos de los fármacos administrados por vía epidural para el control del dolor post cesárea están íntimamente relacionados con las dosis administradas y algunos factores de riesgo en el paciente que lo predisponen a sufrir consecuencias inesperadas o perjudiciales.

Las complicaciones más frecuentes también se asocian a mala manipulación de la técnica. Una de los dispositivos colocados al acceso epidural para la analgesia post cesárea son las bombas de infusión, con una creciente demanda en la actualidad por cuanto permite al paciente controlar el medicamento en función del dolor que siente. Donde la monitorización para su uso, el control de enfermería para su vigilancia y el buen manejo, constituyen factores fundamentales para evitar la presencia de efectos indeseados. 


\section{Contraindicaciones de analgesia epidural post cesárea}

Vol. 3, núm. 2., (2019)

Ivonne Carolina Recalde Tello; Marlon Enrique Allauca Yumiseba; Jhoanna Patricia Puruncajas Maza; Gema Adriana Alarcón Medina

Ochoa Quezada (2013) define la bomba de infusión como aquel dispositivo capaz de inyectar fluidos, nutrientes o fármacos en el sistema circulatorio del paciente. Existen infusiones intravenosas, subcutáneas, arteriales y epidurales. (p. 2)

Las ventajas del uso de una bomba de infusión son: "menor carga de trabajo, mejor analgesia y más seguridad”. (Sociedad de Anestesiología de Chile, 2017)

Sus principales complicaciones son la náusea y el vómito, desde un 4\% (fentanil, meperidina) hasta un 60 o 70\% (neostigmina). Asimismo, la retención urinaria, es un efecto contrario al esperado que se presenta entre un 10 y 15\%. Por otra parte, el prurito constituye uno de los síntomas que se presenta con frecuencia, aunque no referido por las pacientes. El herpes labial, se ha notado en algunos casos con el uso de morfina. La depresión respiratoria es la más temida, afortunadamente es la menos frecuente. (Garza Hinojosa, 2012)

Igualmente, en base a lo anterior se encontró que la mayoría de estos efectos adversos asociados a esta técnica de bomba de infusión se encuentran a su vez relacionados con el tipo de fármaco y la dosis utilizada.

\section{Conclusión}

La analgesia epidural para el control del dolor posterior a una cesárea es un recurso notable que ofrece a la paciente una buena analgesia con menores dosis y efectos secundarios.

Se encontró una pobre literatura relacionada con el estudio de esta técnica a pesar de ser una de las más usadas y con registros de mayores beneficios en todo el mundo. 


\section{Contraindicaciones de analgesia epidural post cesárea}

Vol. 3, núm. 2., (2019)

Ivonne Carolina Recalde Tello; Marlon Enrique Allauca Yumiseba; Jhoanna Patricia Puruncajas Maza; Gema Adriana Alarcón Medina

Las investigaciones disponibles se encuentran orientadas a los efectos de los fármacos administrados, sus dosis y sus tiempos de infusión.

La mayoría de las desventajas son de tipo técnico (manejo del catéter), efecto del fármaco, su dosis y frecuencia de administración y en la vigilancia y control de la vía por parte del personal de enfermería.

Por último, se encontró mediante esta revisión que las ventajas que presenta el uso de esta técnica son mayores que las desventajas en comparación con otras técnicas usadas para la administración de analgesia post cesárea. Asimismo, en base a relación riesgo - beneficio para el paciente se encuentra indicada como la mejor técnica, en términos de mayor duración del analgésico en menores dosis, permite el uso de una amplia gama de fármacos, movilidad precoz del paciente, reducción de estancia y costos hospitalarios, permite la relación temprana de la madre y su hijo y una exitosa y satisfactoria recuperación de la paciente, en líneas generales.

\section{Bibliografía.}

Garza Hinojosa, A. (16 de febrero de 2012). Federación Argentina de Asociaciones de Anestesia, Analgesia Y Reanimación. Recuperado el 08 de agosto de 2019, de https://www.anestesia.org.ar/search/articulos_completos/2/13/357/c.php

Lorca, A. M., \& Calventos, F. (2013). Beneficios de la analgesia epidural en el postoperatorio de cesárea. PARANINFO DIGITAL, 7(19), 1-5. Recuperado el 05 de agosto de 2019, de http://www.index-f.com/para/n19/pdf/066d.pdf

Mayo Clinic. (05 de octubre de 2018). mayoclinic.org/es. Recuperado el 05 de agosto de 2019, de https://www.mayoclinic.org/es-es/epidural-delivery-of-pain-medication/img-20007303

McDonald, J. S., Biing-Jaw, C., \& Wing-Fai, K. (2014). Analgesia y anestesia en obstetricia. En A. H. DeCherney, L. Nathan, N. Laufer, \& A. S. Roman, Diagnósticos y tratamientos gineco obstétricos (11 ed., pág. 1030). no aplica: McGraw-Hill. Recuperado el 06 de agosto de 2019, de https://accessmedicina.mhmedical.com/content.aspx?bookid=1494\&sectionid=98126957 \#undefined 


\section{Contraindicaciones de analgesia epidural post cesárea}

Vol. 3, núm. 2., (2019)

Ivonne Carolina Recalde Tello; Marlon Enrique Allauca Yumiseba; Jhoanna Patricia Puruncajas Maza; Gema Adriana Alarcón Medina

Mille Loera, J. (2018). Analgesia Neuroaxial: ¿Es la mejor alternativa? Anestesia en México, 30(9), 3-9. Recuperado el 07 de agosto de 2019, de http://www.scielo.org.mx/pdf/am/v30n2/2448-8771-am-30-02-3.pdf

Ochoa Quezada, Y. (2013). Bombas de Infusión. Tesis de Grado, Universidad del Azuay, Cuenca. Recuperado el 08 de agosto de 2019, de http://dspace.uazuay.edu.ec/bitstream/datos/2194/1/09656.pdf

Organización Panamericana de la Salud. (2014). paho.org. (O. M. Salud, Ed.) Recuperado el 06 de agosto de 2019, de

https://www.paho.org/nic/index.php?option=com_docman\&view=download\&category_sl ug=datos-y-estadisticas\&alias=696-boletin-informativo-sobre-cesareas $\&$ Itemid $=235$

Organización Panamericana de la Salud. (10 de abril de 2015). paho.org. Recuperado el 07 de agosto de 2019, de

https://www.paho.org/hq/index.php?option=com_content\&view=article\&id=10646:2015la-cesarea-solo-deberia-realizarse-cuando-es-medicamentenecesaria\&Itemid=1926\&lang=es

Ramos, G. E., Ferrer, L. E., Mojica, V. L., \& González, M. (09 de agosto de 2017). Manejo analgésico durante el postoperatorio de. Revista Colombiana de Anestesiología, 45(4), 327-334. Recuperado el 06 de agosto de 2019, de http://www.scielo.org.co/pdf/rca/v45n4/es_0120-3347-rca-45-04-00327.pdf

Ríos, R. (09 de 2017). www.eumed.net. (E. Grupo de investigación (SEJ 309) eumed.net de la Universidad de Málaga, Ed.) Obtenido de www.eumed.net: http://www.eumed.net/libros/libro.php?id=1662

Sociedad de Anestesiología de Chile. (17 de febrero de 2017). sachile.cl. Recuperado el 08 de agosto de 2019, de https://www.sachile.cl/noticias/bolo-intermitente-o-bomba-deinfusion.html

Sukhyanti, K., Kirti, S., \& Bharti, T. (2017). Post-caesarean analgesia: What is new? Indian Journal of Anaesthesia, 61, 200-214. Recuperado el 08 de agosto de 2019, de https://www.ncbi.nlm.nih.gov/pmc/articles/PMC5372400/

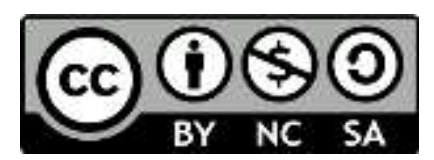

RECONOCIMIENTO-NOCOMERCIAL-COMPARTIRIGUAL

CC BY-NC-SA

ESTA LICENCIA PERMITE A OTROS ENTREMEZCLAR, AJUSTAR Y CONSTRUIR A PARTIR DE SU OBRA CON FINES NO COMERCIALES, SIEMPRE Y CUANDO LE RECONOZCAN LA AUTORÍA Y SUS NUEVAS CREACIONES ESTÉN BAJO UNA LICENCIA CON LOS MISMOS TÉRMINOS. 